\title{
Impact of MDT on leprosy as measured by selective indicators
}

\author{
K JESUDASAN, P VIJAYAKUMARAN, \\ V K PANNIKAR \& M CHRISTIAN \\ Schieffelin Leprosy Research and Training Centre, Karigiri-632 106, \\ S. India
}

\section{Accepted for publication 7 March 1988}

\begin{abstract}
Summary The impact of multidrug therapy (MDT) on the leprosy situation in endemic districts where MDT has been introduced, is studied, using a hypothetical model.

This analysis indicates that there will be significant falls in prevalence rates during the first 5 years, mainly as a result of discharge of cases during screening and due to shortening of the duration of treatment. These changes have to be interpreted with caution. Already some districts like Wardha in India have shown dramatic falls in prevalence rates from 11.1 in 1981 to 1.8 in 1987.

The impact of MDT on disease transmission as measured by decline in incidence rates and case detection rates will, however, be gradual.
\end{abstract}

\section{Introduction}

The National Leprosy Control Programme in India was initiated in $1955 .{ }^{1}$ After nearly three decades of leprosy control using dapsone monotherapy, the strategy was revised in $1982 .{ }^{1}$ The new strategy is based on multidrug therapy (MDT) as recommended by WHO. ${ }^{2}$ MDT was introduced in India on recommendations of the Swaminathan committee ${ }^{1,3}$ in 1983. As of February 1987, 33 endemic districts in India (prevalence 5 or more/1000) have been put under MDT. There are 412 districts in India and leprosy is endemic in 201 of them. Many areas in India thus have 3-5 years experience with MDT.

The initial optimism continues to grow and there is reasonable hope that the leprosy problem can be considerably reduced by the year 2000 . However, it is important to understand the Epidynamics of leprosy in an area where MDT has been introduced. The trends in the leprosy situation, as measured by selected indicators currently being used, have to be interpreted with caution. It is crucial that when looking at these indicators, the actual impact of MDT on transmission of $M$. leprae infection, be differentiated from changes brought about by policy decisions, definitions of indicators and secular trends.

This paper seeks to look at an hypothetical area of one leprosy control unit, where MDT is being introduced at time zero (T0). It seeks to predict what should happen in 3 to 5 years $(\mathrm{T} 0+3, \mathrm{~T} 0+5)$. Since there are considerable differences in interpretation of guidelines on MDT, ${ }^{3}$ use of actual data 
may not be representative. The policy for intake into MDT of paucibacillary and multibacillary patients, criteria for discharge, definitions of indicators and their interpretation vary considerably at the district level.

\section{Methods and materials}

Let us assume the multidrug therapy is introduced in an area of an average leprosy control unit (LCU), with the statistics as follows:

\section{POPULATION DETAILS}

1 Name of LCU

2 Population

3 Area

4 Estimated prevalence

5 No. of estimated cases

6 Estimated incidence rate (annual)

7 New case detection rate (annual)

8 No. of new cases detected each year

Leprad
500,000
1500 sq. km.
$20 / 1000(1981)$
10,000
$2 / 1000$
$1 \cdot 5 / 1000$
750

10,000

2500

7500

$25 \%$

625

1875

2500

$25 \%$

2500

3000

30

2700

NEW CASES PARTICULARS

1 No. of new cases detected per year

750

$1 \cdot 5 / 1000$

75

3 No. of MB cases $(10 \%)$

675

$\begin{array}{ll}5 \text { Deformity rate } & 5 \%-10 \% \\ 6 \text { Proportion of child cases }(40 \%) & 300 \text { cases }\end{array}$

$\begin{array}{ll}5 \text { Deformity rate } & 5 \%-10 \% \\ 6 \text { Proportion of child cases }(40 \%) & 300 \text { cases }\end{array}$

Let us assume that with the above statistics, MDT was introduced in 1981 in the area where dapsone has been used since 1971 . If we take 1981 as 'T0' let us see what will happen to the following selective epidemiological and operational indicators: 1 , prevalence rate; 2 , incidence rate; 3 , deformity rate; 4 , active $\mathrm{MB}$ rate, $\mathrm{PB}$ rate; 5 , positive case rate; 6 , child rate; and 7 , case detection rate. 


\section{DEFINITIONS}

\section{Prevalence rate}

Gross prevalence (GPR): No. of living cases per 1000 population (including released from control, self-healed cases).

Operational prevalence $(O P R)$ : No. of cases requiring treatment or surveillance per 1000 population includes cases under treatment, released from treatment but under surveillance, plus uriregistered cases-sometimes the total is called known cases.

Active prevalence $(A P R)$ : No. of active cases $\mathrm{MB}$ and $\mathrm{PB} / 1000$ population, based on active cases. (It is total $2 \cdot 5+2 \cdot 6$.) It is assumed that prevalence figures are calculated on population actually examined during surveys.

Incidence rate (IR): No. of new cases of leprosy detected per 1000 population per year. The assumption is that if repeated annual population surveys are done, new cases detected among a population previously examined and declared free of leprosy one year earlier, are considered as incident cases. Cases arising from immigrants, births or unexamined population in the previous year, are not included in calculation of incidence rates.

Deformity rate ( $D R$ ): Proportion of cases with visible def ormity (WHO deformity Grade II and III $\left.^{4}\right)$.

$M B$ Rate: Proportion of $\mathrm{MB}$ cases (BL and $\mathrm{LL}$ ) as compared with total cases.

Active Case Rate ( $M B$ and $P B)$. MB, the proportion of $\mathrm{MB}$ cases clinically active or bacteriologically positive as compared with total cases. $\mathrm{PB}$, the proportion of clinically active PB cases as compared with the total cases.

Bacteriologically positive rate (BPR): $\mathrm{PB}$ cases that are skin smear positive in India are grouped along with $\mathrm{MB}$ cases that are positive and are put on the WHO regimen of $\mathrm{MDT}^{3}$ for $\mathrm{MB}$ patients. The proportion of cases that are bacteriologically positive as compared with the total cases under treatment constitute this rate.

Child rate $(C R)$ : Proportion of cases below 15 years of age.

Case detection rate: $(C D R)$ : No. of new cases detected in the area per 1000 population (census). It includes cases detected by all sources including surveys and voluntary reporting.

\section{Results}

CHANGES THAT WILL OCCUR AT T + 1 YEAR-BEFORE MDT IS INTRODUCED

In most LCU an active release from control has not taken place. During the preparatory phase, screening of all cases, PB and MB is done. Intake into MDT is according to the Government of India guidelines. ${ }^{3,5} \mathrm{~PB}$ patients on monotherapy with dapsone are released from control, if: they are classified as indeterminate (Ind), tuberculoid (TT), or borderline tuberculoid (BT), and were skin smears negative at registration; they have completed 3 years of treatment with dapsone; and if they are clinically and bacteriologically inactive as judged by a doctor or a senior non-medical supervisor (NMS).

In patients with $\mathrm{MB}$ leprosy, treatment is continued till clinical and bacteriological inactivity and then a further period of 5 years. In many control programmes however PB and MB patients are treated for much longer periods. It is estimated that the screening phase before MDT is introduced will take 1 year. It is essential to train all cadres of staff to enhance their clinical skills, before screening commences.

\section{CHANGES AT END OF 1 YEAR AFTER SCREENING PERIOD}

If one assumes that only a maximum of $25 \%$ of $\mathrm{PB}$ cases and $\mathrm{MB}$ cases are active: 
The No. of inactive MB cases will be 2500- $625 \quad 1875$

The No. of inactive PB cases will be 7500-1875 5625

Total $\quad \overline{7500}$

Assuming that at least $50 \%$ of the inactive $\mathrm{MB}$ and $\mathrm{PB}$ cases would have completed 7 and 3 years of treatment respectively, $50 \%$ (i.e. 3750 ) cases could be straight away released from control, (remaining $938 \mathrm{MB}+2812 \mathrm{~PB})$.

\section{IMPACT ON PREVALENCE RATE}

If the prevalence rate (OPR) was 20/1000 with 10,000 cases and at screening 3750 cases are released from control, then the prevalence rate would fall to $12 \cdot 5 / 1000,(10,000-3750 / 500,000 \times 1000)$ before MDT is introduced at T0. The prevalence rate would also be considerably influenced by previous definitions of prevalence rates used (see definitions). If the operational prevalence rate was originally used and now the defination is changed to active prevalence rates, and only $25 \%$ of cases are active (i.e. 2500), then the active prevalence rate will be 5/1000. This in itself would show a considerable fall in prevalence rates, even before MDT is introduced. The fall would be greater if gross prevalence rates were originally used.

IMPACT AT THE END OF 1 YEAR

Cases under treatment at beginning of MDT:

$\mathrm{MB}, 625$ active $+50 \% \quad$ (938) inactive 1563

$\mathrm{PB}, 1875$ active $+50 \%(2812) \quad$ inactive 4687

Total $\quad \overline{6250}$

\section{$P B$ cases}

Let us assume that all the PB cases are put under MDT. There are $4687 \mathrm{~PB}$ cases under treatment with 1875 active cases. These PB cases would have received varying durations of treatment with dapsone. However, we will consider that all of them are put under the WHO-PB regimen. At the end of 6 months it is estimated that only $25 \%$ of the active cases will continue to be active, i.e. 469 cases. The remaining 4218 cases, who had had previous dapsone monotherapy, can be released from treatment but are kept under active surveillance for a period of 2 years.

\section{$M B$ cases}

Let us assume that all MB cases (active and inactive) are put under the WHO-MDT regimen. There were $1563 \mathrm{MB}$ cases under treatment and of them 625 were active (bacteriologically, or clinically). At the end of 2 years, the 938 inactive cases would be ready for release from treatment (RFT). At least $\frac{1}{3}$ of the active cases, i.e. 208 with initial BI of 1 and 2 would have become negative and ready for RFT. Thus 1146 cases would be released from treatment but kept under active surveillance for a period of 5 years.

AT THE END OF 3 YEARS THE STATISTICS WOULD BE AS FOLLOWS

Old cases

MB cases under treatment at T0 
MB cases eligible for RFT a t end of 3 rd year (938 inactive +208 , i.e. $\frac{1}{3}$ active cases)

MB cases still requiring treatment (625-208)

PB cases under treatment at T0

New cases

Cases detection rate $1 \cdot 5 / 1000$ population

New cases detected:

$\begin{array}{ll}\text { MB } & 75\end{array}$

PB 675

PB cases eligible for RFT (1st year cases)

PB cases remaining under surveillance ( 2 nd and 3 rd year cases) $675 \times 2$

Total PB cases under surveillance $(469+1350)$

MB cases eligible for RFT ( $10 \%$ of 1 st year cases)

MB cases remaining under treatment ( 2 nd and 3 rd year cases) $75 \times 2$

Total

Total new MB cases under treatment $(150+68)$

Total MB cases under surveillance $(1146+8)$

Total cases under treatment at end of 3 rd year: (old + new)

PB $(50 \%$ of 3 rd year new cases $675 / 2)$

$\mathrm{MB}(417+218)$

Total

At the end of the 3rd year

$\mathrm{MB}$ cases required treatment/surveillance $(1154+218)$

PB cases required treatment/surveillance

Total

Operational prevalence rate $=\frac{3191}{500,000} \times 1000$

Active prevalence rate (635 $\mathrm{MB}+338 \mathrm{~PB}$ active cases)

$\frac{973}{500,000} \times 1000$

Apparent impact of $M D T, 3 r$ year

Prevalence rate (per 1000) T0 After screening $\mathrm{T}+3 \mathrm{yr}$

$\begin{array}{llcl}\text { Operational prevalence rate } & 20 & 12.5 & 6 \cdot 4\end{array}$

Active (APR)

$5 \quad 2$

\section{Incidence rates (IR)}

Based on sample surveys the IR is unlikely to change within $3 \mathrm{yr}$.

\section{Case detection rate}

Will be considerably influenced by intensity of case detection. Since the load of MDT work during 
the first 2 years is high the case detection usually suffers. Overall, no impact on the case detection rate is likely. It is important to retrain staff to improve case detection by such measures as health education aimed at increasing case detection.

\section{Deformity rate}

Among the total cases under treatment and surveillance the deformity rate may in fact go up. The deformity rate is really ratio (proportion), i.e.

$$
\frac{\text { Case with deformity }}{\text { Total case }} \times 100 \text {. }
$$

A large number of PB cases without deformity will be RFCed by the end of the 3rd year, whereas the MB cases with deformity will still be included in the calculation. Depending on the intensity of previous case detection, the deformity rate among new cases will vary. If previous surveys had been done recently deformity rates should fall among new cases.

\section{$M B$ case rate}

The $\mathrm{MB}$ rate will in fact increase as this too is a ratio and the numerator ( $\mathrm{MB}$ cases) will decrease (2500 to 1372), but the total will decrease considerably (10,000 to 3191) as PB cases are released from control. Thus the $\mathrm{MB}$ rate will increase from $25 \%$ to $43 \%$. However, the positive case rate may fall depending on the new MB cases detected.

\section{Child rate}

Among total cases may fall considerably as most of them would be PB and released from control. But the child rate among new cases is unlikely to fall.

\section{STATISTICS AT END OF 5 YEARS}

$\mathrm{MB}$ cases that required treatment of surveillance at end of $3 \mathrm{rd}$ year

PB cases that required treatment of surveillance at end of 3 rd year

Total

All the above P B cases can now be released from control as they would have completed 2 years of surveillance. The 938 inactive $\mathrm{MB}$ cases at $\mathrm{T} 0$ can also be released from control.

New cases

Cases under treatment at end of 3rd year (PB 338+ MB 635)

New cases detected 4 th and 5 th year:

$\mathrm{MB}, 75 \times 2$

$\mathrm{PB}, 675 \times 2$

Total cases requiring treatment or surveillance at end of 5 years: 
Operational prevalence rate: $\frac{2159}{500,000} \times 1000$

Active prevalence rate: $(33 \%) \frac{713}{500,000} \times 1000$

(The positive MB cases would become negative at rate of $10-20 \%$ per year.)

\section{Discussions}

The impact of MDT in 5 years of the prevalence rates as calculated is as follows:

\begin{tabular}{lcccc} 
Prevalence rate & \multicolumn{5}{c}{ After } \\
per 1000 & T0 & screening & $\mathrm{T}+3 \mathrm{Yr}$ & $\mathrm{T}+5 \mathrm{Yr}$ \\
Operational & 20 & 12.5 & 6.4 & 4.3 \\
Active & - & 5 & 2 & 1.4
\end{tabular}

(P) Prevalence $=$ (I) incidence $\times$ (D) duration of the disease. In leprosy the duration of treatment or surveillance is considered the duration a patient is included in prevalence calculations. Then: $\mathrm{I}(2) \times(1-4) \mathrm{D}=\mathrm{P}(2-8)$, where $1-4$ years, is the mean duration of treatment or surveillance.

Thus the impact on the crude prevalence rate after 5 years merely reflects changes in ' $D$ '. One could show a fall in prevalance rate from 20 to 4.3 or 20 to 1.4 depending on definitions of prevalence rates used and policy of release from control prior to MDT. These would not be the true MDT eff ects if one is looking at impact on transmission of $M$. leprae infection and incidence rates of disease.

\section{IMPACT AFTER 5 YEARS OF MDT ON PREVALENCE AND INCIDENCE RATES}

After 5 years as mentioned, the case load is going to decrease considerably once old cases are released from control. Usually when arriving at prevalence rates, 'point prevalence' is calculated, but when incidence rates are arrived at the period incidence is calculated. In such instances $\mathrm{D}=$ =duration of disease, if taken as duration of treatment of leprosy (excluding period of surveillance), the incidence rate can turn out higher than the prevalance rates.

At the end of 5 years the figures are as follows for the total number of cases requiring treatment or surveillance:

\begin{tabular}{lrrr}
\hline & Treatment & Surveillance & Total \\
\hline PB & $675 / 2=338$ & $1350-338=1012$ & 1350 \\
MB & $75 \times 2=150$ & $1372-938=434$ & 584 \\
\hline Total & 488 & 1446 & 1934 \\
\hline
\end{tabular}

Approximately $338 \mathrm{~PB}$ cases will require treatment. This is because the other 1012 cases are in the 2 years surveillance phase.

Assuming that 675 new PB cases and 75 new MB cases are detected each year, at the beginning of the 6th year the only PB cases under treatment will be those detected in the second half of the previous (5th) year. During the year these cases, plus most of the cases detected during the first half of the 6th year, will be released from treatment. Thus at the end of the year onlyhalf the new PB cases detected will be under treatment, plus the 75 new MB cases. During the 6th year closing figures will be: 


$\begin{array}{clr}\text { Old cases under treatment } & \text { PB } & 338 \\ \text { (5 years MB cases) } & \text { MB } & 375 \\ & & 713 \\ \text { New cases detected 6th year } & \text { PB } & 675 \\ & \text { MB } & \underline{75} \\ & & \underline{750}\end{array}$

Based on the above figures, the cases under treatment only at end of the 6th year will be: $\mathrm{MB} 375+338 \mathrm{~PB}$ cases $=713$. The point prevalence rate calculated on cases under treatment only will be: $713 / 500,000 \times 1000=1 \cdot 4$. The active point prevalence will be: $(40 \%$ of $713=$ $285 / 500,000 \times 1000)=0 \cdot 57$. The case detection rate $(C D R)$, however, will be $750 /$ $500,000 \times 1000=1 \cdot 5$. Thus the CDR will be 2.63 times the prevalence rate!! The incidence rate will still probably be $2 / 1000$.

In Wardha, a MDT district in India, the active prevalence rate (APR) is $1 \cdot 8 / 1000$ and the case detection rate (CDR) is 2.6 in 1986, similarly in Vijinagaram the APR $=2 \cdot 8$ and the CDR is $2 \cdot 2 .{ }^{6}$

The calculation $\mathrm{P}=\mathrm{I} \times \mathrm{D}$ no longer holds good when, in a chronic disease, ' $\mathrm{D}$ ' becomes less than 1 year. When targets for case detection are calculated this has to be borne in mind. These trends in MDT districts also indicate that the concepts of incidence and prevalence have to be reviewed.

There is, however, a considerable reduction in case load and fall in prevalence rates. In most MDT districts in India this is seen-Wardha, a fall in APR from $11 \cdot 1$ to $1 \cdot 8$, Purulia $19 \cdot 2$ to $7 \cdot 7$, Srikakulam 18 to $2 \cdot 6 .{ }^{6}$ This reduction has to be viewed with caution. There may be a large number of disabled and deformed leprosy patients now removed from the statistics and declared 'cured', who may still need considerable supportive care in the years to come- 'Care After Cure'.

Assuming that the incubation period of leprosy is 3-5 years one can begin to see the effects of MDT on transmission, as measured by incidence rates only after 5 years. An early marker, would be measurement of $M$. leprae infection using serological tests. The incidence rate as well as the case detection will probably decline after 5 years.

Changes in the def ormity rate, incidence rate a mong contacts, the child rate and the $\mathrm{MB}$ rate will occur among the new cases, assuming intensive case detection continues and the backlog of old cases have been detected and treated.

It was initially suggested ${ }^{7}$ that $80 \%$ of estimated cases would have to be detected before MDT is to be introduced. This would mean that all the new cases detected would be put on dapsone monotherapy, till MDT was introduced. Hence the current thinking is that MDT be introduced irrespective of what proportion of estimated cases have actually been detected. But during the first 2 years of MDT, the backlog of cases would have to be detected in an intensive case detection programme. The impact of MDT will be considerably influenced by the gap between the estimated cases and actual cases under treatment.

An extremely interesting article on the 'Epidemiology and decline of leprosy in Asia'8 suggests that, using composite data from East Asia, the decline in new cases had already begun in the 1960s. The fall, however, has been gradual up to the year 1980 prior to MDT. Skinsnes ${ }^{8}$ commenting on the East Asia model indicates that it would take 25 years after the peak in the new case detection rate, for leprosy to come down to $25 \%$ of its peak.

Assuming that the MDT effect is more pronounced and rapid, one would assume that the declines in the new case detection in MDT districts would be apparent after 5 years of MDT.

The impact of MDT would probably be earlier in low prevalence areas, if a high proportion of estimated cases are put under effective chemotherapy.

At the managerial level, however, one has to keep in mind that: 
The impact of MDT will mainly be on reduction in the case load for the first 5 years.

This impact is significant, but interpretation of the falls in prevalence rates cannot imply fall in transmission.

Incidence rates will probably be affected only after 5 years.

Discharge of a large number of cases from statistical formulation should not make one forget patient's long term rehabilitative needs.

Targets based on prevalence rates alone could be misleading.

The fall in prevalence rates is useful managerially in planning resource allocation.

As active case finding is likely to suffer during the initial period after the introduction of MDT, measures to enhance voluntary reporting by means of health education should be encouraged.

It has also got to be understood that the impact on the trends in leprosy, as measured by selected indicators, will be considerably influenced by:

Definitions of indicators and their interpretation.

Efficiency of programme implementation before and after MDT.

Adequacy of population coverage, case detection, case holding and the service delivery components.

Initial prevalence and incidence rates.

When one looks at the possible impact of MDT on theleprosy situation by the year 2000, there is considerable cause for optimism. This optimism, however, should be guided by the knowledge of the Epi-dynamics of leprosy in an MDT area.

\section{Acknowledgments}

We wish to thank the Danish International Development Agency, Dr G D Georgiev and the Government of India especially, Dr C K Rao for organizing numerous workshops in the areas of evaluation and monitoring. Participation in these workshops and interaction with colleagues from many MDT districts, helped develop the ideas that have been formulated in this paper. We also wish to thank Mr N Christopher and Mr C Lewis for their secretarial help.

\section{References}

1 Government of India, Ministry of Health and Family Welfare, New Delhi-Report on the Working Group of Eradication of Leprosy, 1982; p. 2.

2 WHO Study Group. Chemotherapy of leprosy for control programmes. Technical Report Series No. 675. WHO: Geneva, 1982.

${ }^{3}$ Government of India-Leprosy, Guidelines in case detection, treatment, follow-up and reporting, 1985; Published by Leprosy Division, DGHS, Nirman Bhavan, New Delhi, p 6.

4 WHO Study Group. Report on disability grading scale. p 28. Technical Report Series No. 459. WHO: Geneva, 1970.

5 Government of India-Guidelines on Multidrug, treatment in endemic districts, NLEP (1986). Published by Leprosy Division, DGHS, Nirman Bhavan, New Delhi, p 12 (revised edition published in 1987).

${ }^{6}$ Leprosy, NLEP in India. Report of the Second Independent Evaluation of NLEP. 1987; Published by Leprosy Division, Nirman Bhavan, New Delhi.

${ }^{7}$ Evaluation and Monitoring in the National Leprosy Eradication Programme-1986. Report prepared by the Danish International Development Agency, No. 7, Golf Links, New Delhi.

8 Skinsnes OK. Epidemiology and decline of leprosy in Asia. Int J Derm, 1983; 22 348-67. 
NEWS AND NOTES

\section{Armadillo survey: Universities of Oxford and Minas Gerais, Brazil 1988}

The explanatory brochure for this project reads as follows:

The Serra da Canastra National Park lies in the province of Minas Gerais, S.E. Brazil. It is a beautiful 71,525 ha area of grassland plateau at $1400 \mathrm{~m}$ above sea level, and is a rich source of wildlife, dominated by armadillos and their relatives, the anteaters.

It is hoped that this joint project between the Universities of Oxford and Minas Gerais will enhance AngloBrazilian relations. The students of both nationalities should, through shared experience with contemporaries of a different cultural background, gain a greater mutual understanding.

The Survey aims to continue the work of Dr Christiane Encarnação who, in her Ph.D. thesis, investigated the social behaviour of the 6 species of armadillo in the Park. A rudimentary population census will be carried out for each species, together with a general study of their behaviour and ecology and an investigation of their ectoparasites. Studies will be centred around the 6-banded armadillo, Euphractus sexcinctus.

There are, in all, 20 species of armadillos, which range in length from $10 \mathrm{~cm}$ (the pink fairy armadillo, Chlamyphorus truncatus, of Argentina) to $1 \mathrm{~m}$ (the giant armadillo, Priodontes maximus, thinly scattered over most of S. America). They principally feed on ants and live in burrows.

The 6-banded armadillo (Euphractus sexcinctus), the main study subject, is about $40 \mathrm{~cm}$ long. Unusually, it is largely diurnal. Although not a widely distributed species, and hence little studied, it is the commonest in the Park.

Other species to be seen in the Park are: 'the naked-tailed armadillos ( 2 species-Cabassous unicinctus squamicaudis and Cadassous tatouay); the long-nosed armadillos (2 species-Dasypus novemcinctus and Dasypus septemcinctus); and the giant armadillo (Priodontes maximus).

Armadillos will be caught in humane traps, as used by Tracy Carter and Christiane Encarnação. ${ }^{1}$ These may be acquired cheaply in Brazil. The animals will then be tracked by a combination of the following methods.

Spool-and-line tracking

Spool-and-line tracking devices essentially consist of a fixed spool of thread, with a range of up to $2300 \mathrm{~m}$, enclosed in a plastic sheath. This is attached to the study animal, the thread pays out freely, and the trail may subsequently be followed to the burrow. This technique, developed by Dr M A Miles of the London School of Hygiene and Tropical Medicine $e^{4,5}$ provides information on distances travelled at night, home, range, size and burrow type and location. It complements radiotracking and is advantageous in that it is inexpensive and allows the tracking of several animals simultaneously and continuously.

Radio-tracking

Betalights - details are available from the address below.

Each armadillo caught will be examined for ectoparasites, which will be identified by an expert at the Federal University of Minas Gerais.

\section{Bibliography}

1 Carter TS, Encarnação CD. Characteristics and use of burrows by four species of armadillos in Brazil. $J$ Mammal, 1983; 64: 103-8.

${ }^{2}$ Greegor DH Jnr. Preliminary study of movements and home ranges of the armadillo (Chaetophractus vellerosus). J Mammal, 1980; 61: 334-5.

${ }^{3}$ A handbook on biotelemetry and radio-tracking. Macdonald DW, Amlaner CJ (eds.) Pergamon Press, 1980.

4 Miles MA. A simple method of tracking mammals and locating triatomine vectors of Trypanosoma cruzi in the Amazon forest. Am J Trop Med Hyg 1976; 25: 671-4.

${ }^{5}$ Miles MA, de Souza AA, Popoa MM. Mammal tracking and nest location in Brazilian forest with an improved spool-and-line device. J Zool Lond, 1981; 195: 331-7.

6 The evolution and ecology of armadillos, sloths and vermilinguas. Montgomery GG (ed.) Smithsonian Inst. Press, 1985.

7 Walker EP. Mammals of the world (3rd ed.). John Hopkins University Press, Baltimore and London, 1975. 